\title{
Multichannel esophageal signals to monitor respiratory rate in preterm infants
}

\author{
Corine Bürgin ${ }^{1}$, Patrizia Simmen ${ }^{1}$, Nishant Gupta ${ }^{2}$, Lilian Suter ${ }^{1}$, Samuel Kreuzer ${ }^{2}$, Andreas Haeberlin ${ }^{3,4}$, Sven M. Schulzke ${ }^{1}$, \\ Daniel Trachsel ${ }^{1}$, Thomas Niederhauser ${ }^{2}$ and Kerstin Jost (iD ${ }^{1,5} \dot{\Phi}^{\prime}$
}

(c) The Author(s) 2021

BACKGROUND: Apnea of prematurity cannot be reliably measured with current monitoring techniques. Instead, indirect parameters such as oxygen desaturation or bradycardia are captured. We propose a Kalman filter-based detection of respiration activity and hence apnea using multichannel esophageal signals in neonatal intensive care unit patients.

METHODS: We performed a single-center observational study with moderately preterm infants. Commercially available nasogastric feeding tubes containing multiple electrodes were used to capture signals with customized software. Multichannel esophageal raw signals were manually annotated, processed using extended Kalman filter, and compared with standard monitoring data including chest impedance to measure respiration activity.

RESULTS: Out of a total of $405.4 \mathrm{~h}$ captured signals in 13 infants, 100 episodes of drop in oxygen saturation or heart rate were examined. Median (interquartile range) difference in respiratory rate was $0.04(-2.45$ to 1.48$) / \mathrm{min}$ between esophageal measurements annotated manually and with Kalman filter and -3.51 ( -7.05 to -1.33$) /$ min when compared to standard monitoring, suggesting an underestimation of respiratory rate when using the latter.

CONCLUSIONS: Kalman filter-based estimation of respiratory activity using multichannel esophageal signals is safe and feasible and results in respiratory rate closer to visual annotation than that derived from chest impedance of standard monitoring.

Pediatric Research (2022) 91:572-580; https://doi.org/10.1038/s41390-021-01748-4

\section{IMPACT}

- Using extended Kalman filtering applied to multichannel esophageal signals enables estimation of respiratory rate in preterm infants under clinically meaningful circumstances.

- Conventional chest impedance measurements are known to be prone to motion artifacts, which makes apnea detection unreliable. Therefore, indirect measures as oxygen desaturation are commonly taken as indicator for such events. In contrast, esophageal signals benefit from a stable electromechanical interface, making them less prone to artifacts. Integration of esophageal electrodes into a clinically required feeding tube is an additional benefit of the applied technique.

- Less artifacts in respiratory rate measurement would improve apnea detection and hence patient care.

\section{INTRODUCTION}

Apnea of prematurity is a well-described condition in preterm infants. ${ }^{1-3}$ Various apnea definitions exist, commonly referring to a cessation of breathing of at least $15-20 \mathrm{~s}$, potentially being associated with hypoxia and/or bradycardia. ${ }^{4,5}$ Subgroups such as central, obstructive, or mixed apnea are further distinguished.,
Importantly, prolonged apnea episodes leading to considerable oxygen desaturation $(<80 \%)$ increase the risk of death or longterm neurodevelopmental impairment in preterm infants. ${ }^{7,8}$

An unresolved problem in daily clinical care on a neonatal intensive care unit (NICU) is the unreliable detection of respiratory rate (RR) with standard monitoring systems using chest impedance $(\mathrm{Cl}) .{ }^{9} \mathrm{Cl}$ is known to be prone to motion artifacts, which may lead to false positive or false negative breath detection. ${ }^{10}$ Consequently, in case of apnea, an audible alarm is often not triggered by the $\mathrm{Cl}$ monitor, but rather by accompanying monitoring systems observing oxygen saturation $\left(\mathrm{SpO}_{2}\right)$ or heart rate (HR), i.e., when associated hypoxia or bradycardia occur.

To overcome the limitations of $\mathrm{Cl}$, surface diaphragmatic electromyography (EMG) has been proposed to detect RR in neonates. ${ }^{11}$ Surface EMG might be superior in differentiating the apnea subtypes; however, similar susceptibility of surface EMG to motion artifacts as $\mathrm{Cl}$ have been shown. ${ }^{12}$ Additionally, EMG as well as $\mathrm{Cl}$ rely on surface skin electrodes, which may harm the fragile skin of preterm infants with a subsequently increased risk of infection. ${ }^{13,14}$

Neurally adjusted ventilatory assist (NAVA) was developed to measure diaphragmatic activity and synchronize mechanical ventilation with the patients' own breathing effort. ${ }^{15,16}$ NAVA uses commercially available gastric feeding tubes equipped with multiple

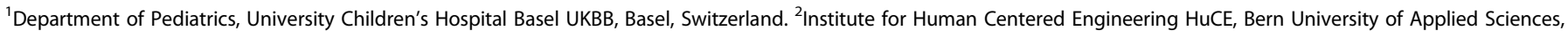

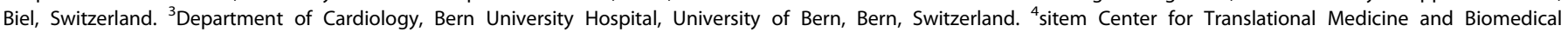
Entrepreneurship, University of Bern, Bern, Switzerland. ${ }^{5}$ Department of Women's and Children's Health, Karolinska Institutet, Stockholm, Sweden. ${ }^{凶}$ email: Kerstin.jost@ki.se 
electrodes, i.e., the Edi tube (Maquet, Getinge Group, Solna, Sweden) to detect diaphragmatic activity of the patient to trigger the ventilation. If esophageally detected respiration can be used safely and effectively for synchronization of non-invasive respiratory support in preterm infants is not yet determined due to limited data, ${ }^{17}$ although its feasibility has been demonstrated before. ${ }^{18}$

In a previous publication, we demonstrated reliable HR detection in non-ventilated preterm infants using multichannel esophageal signals from such a feeding tube. ${ }^{19}$ Based on the same preterm dataset, the present study aims to estimate RR from multichannel esophageal signals in non-ventilated infants. Respiratory activity is dominated by diaphragmatic motion and causes esophageal electrodes to move, as well, which manifests as baseline wander in esophageal signals. Similarly, baseline wander may result from cardiac motion and esophageal peristalsis. ${ }^{19}$ Due to overlapping frequency spectra of these three components of baseline wander, ${ }^{20}$ and further complications from catheter motion artifacts, e.g., due to coughing, simple linear (frequency-based) filters are not applicable to reliably isolate respiratory motion. ${ }^{21}$ For this purpose, model-based filtering, such as Kalman filters, might provide a solution. Kalman filters have successfully been applied, e.g., to denoise multichannel electrocardiogram (ECG) signals with strong interferences or RR estimation from combined surface ECG and photoplethysmography measurements. ${ }^{22-24}$

\section{Hypothesis}

We hypothesize that (i) detection of RR by model-based processing of multichannel esophageal signals is safe and feasible in non-ventilated infants during daily clinical practice in the NICU and (ii) derived $\mathrm{RR}$ is comparable to $\mathrm{Cl}$ respiratory monitoring during clinically meaningful events including oxygen desaturation obtained from pulse oximetry and bradycardia detected by ECG.

\section{METHODS}

\section{Study design and subjects}

This is a prospective single-center observational study, conducted at the University Children's Hospital Basel, Switzerland. Between July 2015 and April 2016, we prospectively included infants with a postmenstrual age between 32 and 42 weeks, after their first week of life. Inclusion criteria were a signed informed consent of the parents, need of a gastroesophageal feeding tube, and presumed stay in the NICU for $>5$ days. Exclusion criteria were major congenital malformations, need of vasoactive drugs, and endotracheal ventilation. Demographic and clinical data from the perinatal period to first discharge home or transfer to another hospital was collected. Documentation of possible adverse events such as tube misplacement or secondary dislocation as well as observation of skin irritation at place of external fixation was noted for all participants. All patient data were stored in a coded format.

The study was approved by the Ethical Committee of Northwestern and Central Switzerland (EKNZ: 2015-136) and was performed in accordance with the principles of the Declaration of Helsinki. The study was registered at ClinicalTrials.gov (identifier NCT02501512).

\section{Measurement set-up and protocol}

Before onset of the study, the normal feeding tube was replaced by an Edi catheter conventionally used in conjunction with NAVA ventilation. This polyurethane-based tube is equipped with ten stainless steel electrodes placed at the distal end with an inter-electrode distance of $6 \mathrm{~mm}$. The most proximal esophageal electrode (electrode \#1) was used as ground. The second most proximal electrode (\#2) served as reference. We then formed bipolar leads, referred hereafter as eECGm -2 , with $m$ depicting the more distal electrodes ( $\# 3$ to \#10), providing $n=8$ esophageal leads. Esophageal signals, hereafter called NEO (Neonatal Esophageal Observation) signals, were captured with customized software built for this study based on MATLAB (The MathWorks', Inc., Natick). Correct placement of the electrode was ensured using a graphical user interface of the software depicting esophageal ECG signal with visible amplitudes and correct feeding capability. The esophageal signals were acquired using a dedicated recorder (g.USBamp, g.tec medical engineering GmbH, Schiedlberg, Austria). The recorder amplified the unfiltered signals using direct current (DC) coupling, an input range of $\pm 250 \mathrm{mV}$, and a sampling rate of $4800 \mathrm{~Hz}$.

In addition to the esophageal electrodes, three pre-gelled surface electrodes designed to be used in preterm infants (Multi Biosensors Inc., TX) were attached to the infant's thorax. The additional bipolar ECG, necessary for later synchronization of the NEO signals with the standard monitoring ECG, was amplified and simultaneously digitized in the same way as the esophageal leads and stored in the customized software.

Standard monitoring data (hereafter called NICU) were stored and synchronized to $N E O$ signals to ensure comparison with state-of-the-art technology. The data extraction from bedside NICU monitor screens (Philips IntelliVue MX, 700, Philips, Amsterdam, the Netherlands) was enabled using the commercially available software (ixTrend 2.0 Express, ixcellence $\mathrm{GmbH}$, Wildau, Germany). The NICU output included the following signals and the corresponding numeric series: surface ECG and $\mathrm{HR}, \mathrm{Cl}$ and $\mathrm{RR}$, and $\mathrm{SpO}_{2}$ level. An overview of the study setting can be seen in Fig. 1.

Briefly, we performed NEO and NICU measurements on 5 consecutive days in each study participant. On the first and last day, measurement duration was $24 \mathrm{~h}$; on days $2-4$, periods of $3 \mathrm{~h}$ were recorded. A detailed description of the study set-up, measurement protocol, and NEO signal processing has been published elsewhere. ${ }^{19}$

\section{Signal pre-processing}

To be able to compare NEO and NICU signals, we first performed resampling of NICU data to the sampling frequency of NEO data and

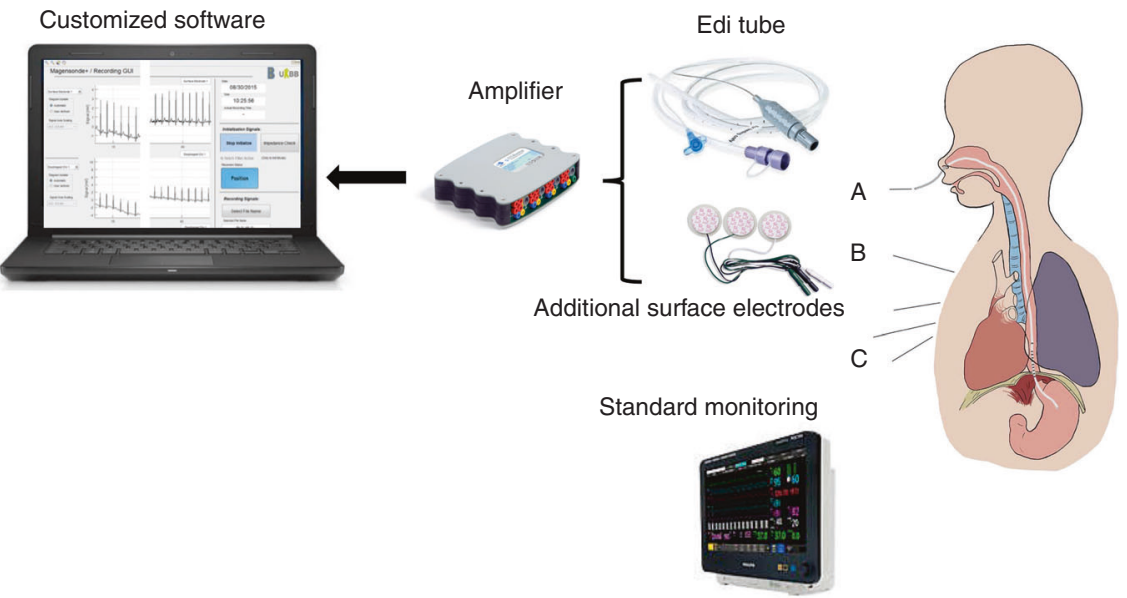

Fig. 1 Overview of study setting. (A) Multichannel Neonatal Esophageal Observation (NEO) signal acquisition using the Edi tube, (B) simultaneous recording of surface electrocardiography. (C) NICU standard monitoring, including electrocardiography to measure heart rate, chest impedance measurement for the detection of respiratory rate, and pulse oximetry to measure peripheral oxygen saturation. 

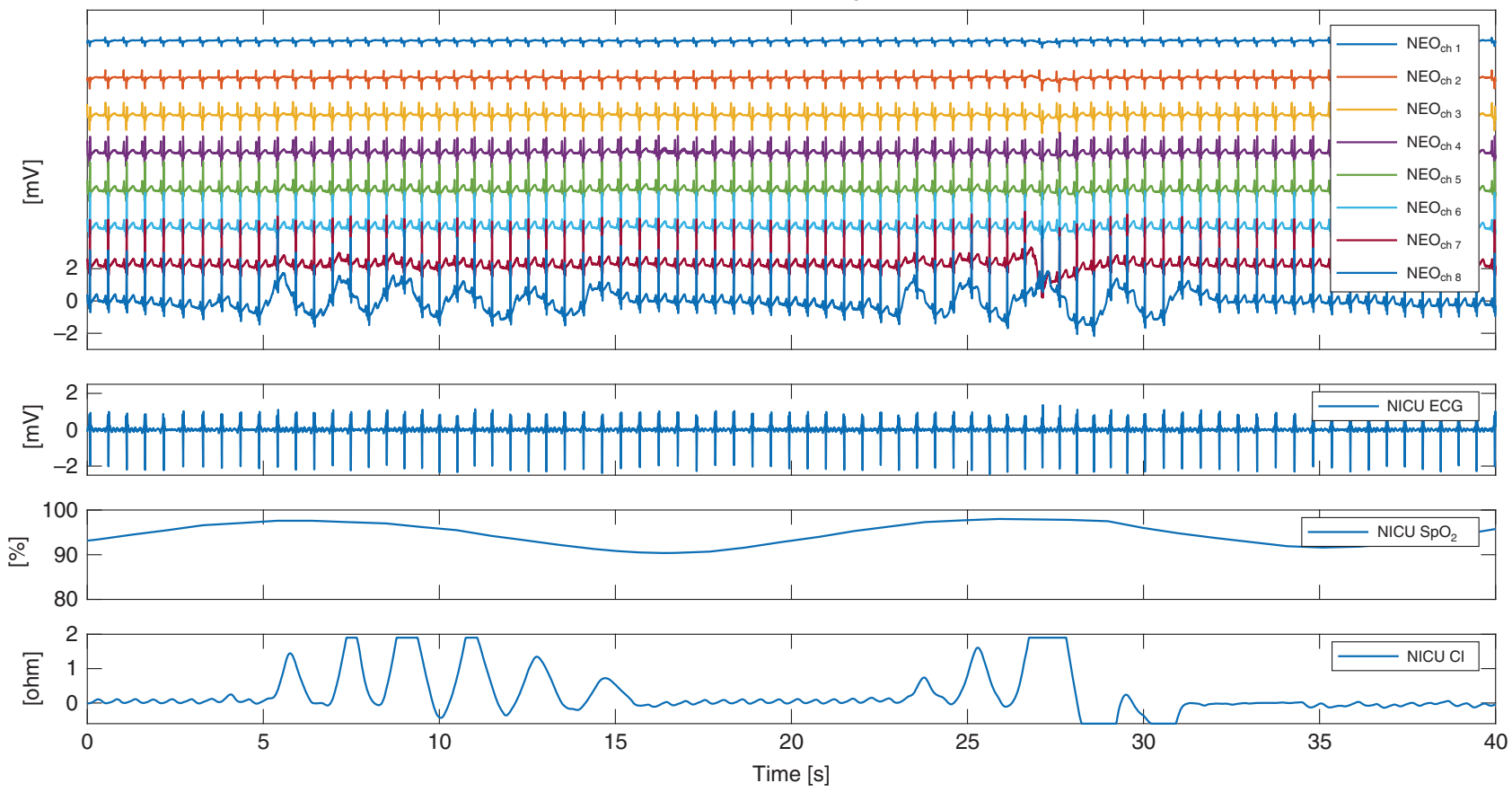

Fig. 2 Example of NEO and NICU signals during a 40-s period. First panel: raw signal of eight esophageal leads (NEO channel 1: most proximal, channel 8: most distal lead), second panel: surface NICU electrocardiography (ECG) signal, third panel: $N I C U$ oxygen saturation (SpO ${ }_{2}$ ) signal, fourth panel: NICU chest impedance (Cl) signal.

synchronized them based on QRS-peak detection from both sources to eliminate possible drift of signals. A notch-filter $(50 \mathrm{~Hz}$, bandwidth 1.4 $\mathrm{Hz}$ ) was applied to the raw NEO data to suppress power-line interference.

An example of synchronized data from NEO and NICU signals can be seen in Fig. 2 for the whole set-up, as well as in Figs. 1 and 2 of the Supplementary Material for good and bad signal quality, respectively.

\section{Data processing and labeling}

Measurement periods with clinically relevant events, namely, bradycardia (values $<80$ beats per minute over at least $5 \mathrm{~s}$ ) or drop in $\mathrm{SpO}_{2}$ (values $<88 \%$ over a period of at least $5 \mathrm{~s}$ ) based on the NICU numerics data output were automatically detected. We chose a short duration of hypoxia and bradycardia, as we were studying a rather healthy population of moderately preterm infants, and because many apneas are known to be of shorter duration than $20 \mathrm{~s}^{5}$ For each event, a window of at least $2 \mathrm{~min}$ (1 min before onset of bradycardia and/or oxygen desaturation until $1 \mathrm{~min}$ after resolution of the event) was analyzed.

Out of the 964 detected bradycardia and/or drop in desaturation events, 100 were selected without prior knowledge of patient characteristics or signal quality. A trained physician (C.B.) visually labeled esophageal respiratory signal in a customized software, not knowing when exactly in the segment the bradycardia/oxygen desaturation occurred and neither which condition was present. The labeling process and comparison of outcomes is illustrated in Fig. 3 and was performed as follows: out of the eight esophageal channels, the labeling physician chose the channel with the strongest respiration signal after visual inspection of an overview of all channels. The labeling of the respiratory waves was subsequently done on a zoomed window of $20 \mathrm{~s}$ of the selected channel. In areas with a doubtful signal, all other channels could have been considered.

Two auxiliary lines representing the 25th and 75th percentile of the median signal amplitude for each signal trace as well as the surface ECG were additionally visible on request to distinguish respiratory activity from cardiac motion, esophageal peristalsis, and catheter motion artifacts. The synchronized NICU respiration signal was displayed as well (see also Figs. 1 and 2 of the Supplementary Material).

All labeled segments were divided by the physician's impression of the esophageal respiratory signal quality into one of the following groups: good, medium, and poor signal quality.

\section{Extended Kalman filter}

In parallel to the visual annotation, the Kalman filter was applied to the same segments of clinically relevant events. The idea behind a Kalman filter is to estimate hidden time-varying process parameters based on timecorrelated observational measurements. In the case of respiratory activity, neither the electrical activation nor the motion of the diaphragm is directly measurable with esophageal signals. The Kalman filter, however, estimates RR based on the surrogate marker of low-frequency electrode motion manifested as baseline wander. The baseline wander, particularly pronounced in the distal channels placed close to the diaphragm, correlates well with the respiration activity as shown in Fig. 2. To extract the RR from the multichannel esophageal signals, the respiration was modeled as a sinusoidal signal with a common time-varying frequency but independent amplitudes for each channel. Using the extended Kalman algorithm, instantaneous values of the frequency and subsequently the phase angle (frequency integrated with time) were estimated for each segment. Respiration rates $R R+$ and $R R-$ were then calculated from the time differences between $0^{\circ}$ and $180^{\circ}$ phase crossings, respectively. The NEO RR was finally estimated by averaging RR + and RR - and applying a moving mean filter with a window length of $5 s$ to attenuate model inaccuracies. No prior information about the location of the esophageal channels most sensitive to respiration activity was needed, as information from all channels were considered simultaneously. Details of the developed model and the extended Kalman algorithm will be published separately.

\section{Inter-rater reliability}

To assess inter-rater reliability, we selected 5,8 , and 7 segments of poor, medium, and good signal quality, respectively, to be labeled by a second physician (K.J.) who was blinded to the results of the first labeling process.

\section{Statistical analysis}

Statistical analysis was performed with MATLAB. For each segment of 2 min duration, we took the manual labeling of NEO respiratory signal by the first physician as reference. For each segment, median and interquartile range (IQR) of the difference from the reference RR (in units breaths per minute (bpm)) for the NEO derived by Kalman filter and NICU derived by $\mathrm{Cl}$ were obtained and were defined as deviation and variability, respectively. This result can be interpreted for both deviation and variability 


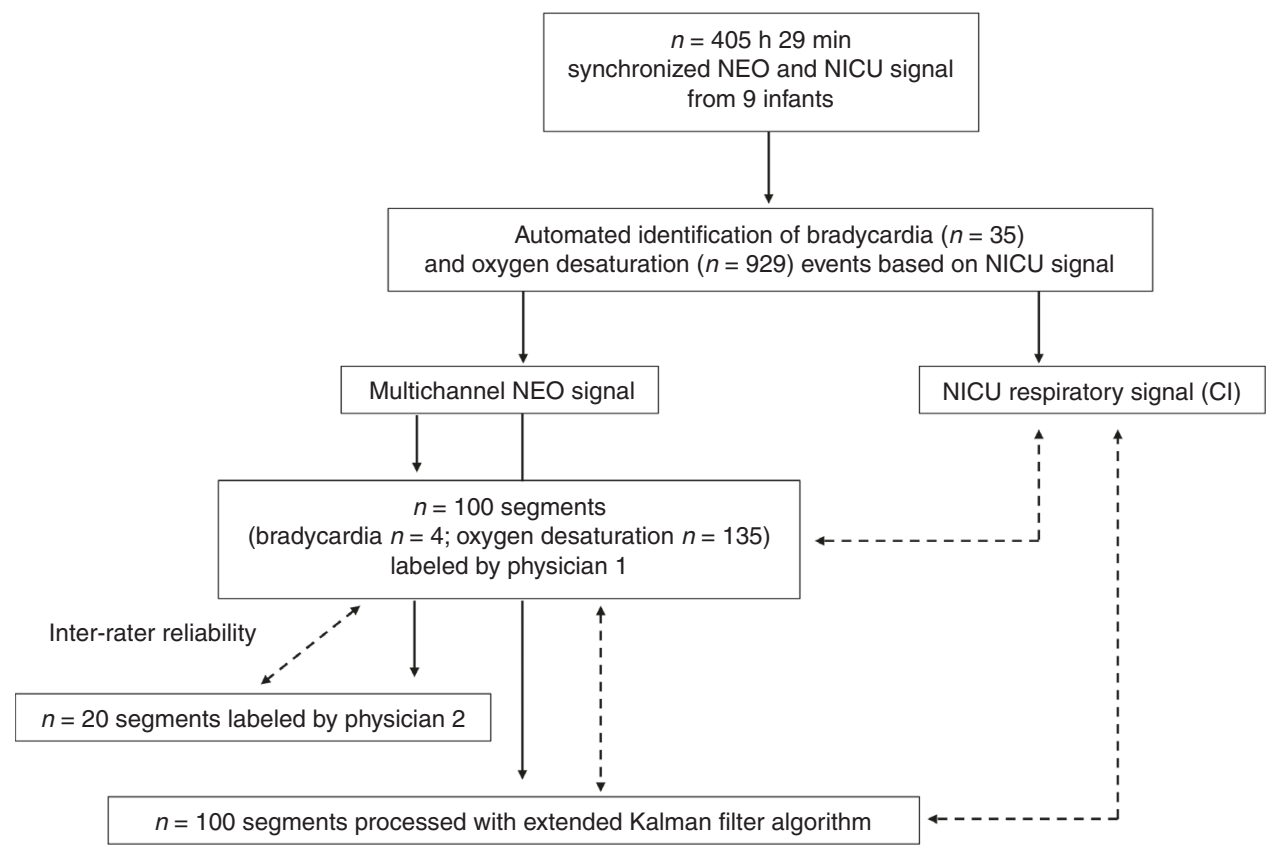

Fig. 3 Labeling of 100 randomly chosen clinically relevant events by physicians was done by visual inspection of multichannel $\mathbf{N E O}$ signals. Solid lines: flow of data through different assessment steps; dotted lines: comparison of respiratory rate by different sources, using Wilcoxon signed-rank test and Spearman's rank correlation coefficient for inter-rater reliability. Cl chest impedance, NEO Neonatal Esophageal Observation, NICU Neonatal Intensive Care Unit.

as 0 to be the perfectly matching signal with less agreement the further away from 0 . We compared the distribution of deviation and variability of the difference of RR from the reference for both methods over 100 segments. For variability, a cut-off of $15 \mathrm{bpm}$ was defined to be clinically meaningful, since it is lower than the normal range of RR in neonates (40-60 bpm) but still of clinical relevance. To illustrate the level of agreement between the different measurement techniques, we displayed the results in a Bland Altman plot. Each observation point in the plot shows the mean RR of a time window of $5 \mathrm{~s}$. The short window length was chosen to account for periodic breathing episodes which would get averaged out with larger windows.

Statistical significance was tested using a Wilcoxon signed-rank test, and adjustment for multiple comparison was done using a Bonferroni-Holm correction. We assumed a significance level alpha of $<0.05$ after the correction.

Inter-rater reliability was assessed using Spearman's rank correlation coefficient, which can take a value from -1 to $+1 ;-1$ meaning opposite agreement (increasing vs. decreasing RR), 0 meaning no association between the two raters, and +1 describing perfect agreement. Values significantly higher than 0.5 are considered relevantly correlated and of $\geq 0.7$ are considered as highly correlated. We further assessed the dependency of inter-rater reliability on visually labeled NEO signal quality by grouping comparison based on good, medium, and poor signal quality. Demographic factors of the subgroup of infants contributing to the selected signal episodes were compared with the overall study population using Mann-Whitney $U$ test for continuous variables and Fisher's exact test for comparison of categorical variables.

\section{RESULTS}

\section{Study population and measurements}

Table 1 of the Supplementary Material shows an overview of demographics of study participants. In total, 60 measurements performed in 13 infants ( $n=7$ male) with a mean ${ }^{14}$ gestational age of 33.0 (28.9-35.1) weeks and birth weight of 1621 $(970-2340) \mathrm{g}$ were considered. We finally included a total of 31 measurements of $405.4 \mathrm{~h}$ duration performed in 9 infants for further signal analysis after excluding 29 measurements for reasons including transfer of the infant to another hospital $(n=$ 5), transfer to another ward with different standard monitoring system ( $n=16)$, inadequate signal quality or interrupted sampling $(n=2$ for NEO; $n=6$ for NICU). No adverse events were noted throughout the study.

Within these 31 measurements, 964 clinically relevant episodes of bradycardia ( $<80$ beats per minute over $>5 \mathrm{~s})$ or oxygen desaturation $\left(\mathrm{SpO}_{2}<88 \%\right.$ over $\left.>5 \mathrm{~s}\right)$ were detected. Mean (standard deviation) duration of bradycardia and oxygen desaturation episodes was $16.6(4.7)$ and $27.3(45.9) \mathrm{s}$, respectively, with lowest values of 62.3 (2.5) beats per minute for HR and 81.3 (6.1)\% for $\mathrm{SpO}_{2}$. In the randomly selected $n=100$ segments of $N E O$ signal, 8 of the 9 infants with complete datasets were represented, 1 infant coincidently fell out. These segments consisted of 23 episodes with good, 54 with medium, and 23 with poor signal quality, as characterized by visual inspection. Characteristics of overall study population and infants contributing to the finally selected episodes did not show any significant differences (see also Table 1 in the Supplementary Material).

\section{Main findings}

RR was successfully extracted by the Kalman filter in the selected segments. When all 100 segments were considered, median (IQR) RR deviation from manually labeled NEO reference was $0.04(-2.45$ to $1.48, p=1.0) \mathrm{bpm}$ for Kalman filter and $-3.51(-7.05$ to -1.33 , $p<0.001$ ) bpm for NICU, respectively (see Fig. 4a). RR variability within the segments when compared to visually labeled reference was significantly lower in NEO Kalman-derived RR with median (IQR) 10.29 (7.46 to 13.56) bpm vs. NICU derived RR with median (IQR) 18.09 (13.53 to 21.83) bpm, $p<0.001$ (see Fig. 4b).

The narrower concentration of the points around the $x$-axis in the Bland Altman plot (Fig. 5) represents the smaller deviation and variability of RR NEO from visual annotation compared to NEO analyzed by Kalman filter (Fig. 5b) than when compared to NICU (Fig. 5a).

\section{Subgroup analysis based on signal quality}

Subgroup analysis by visually categorized esophageal signal quality did not show significant differences in NEO RR using 

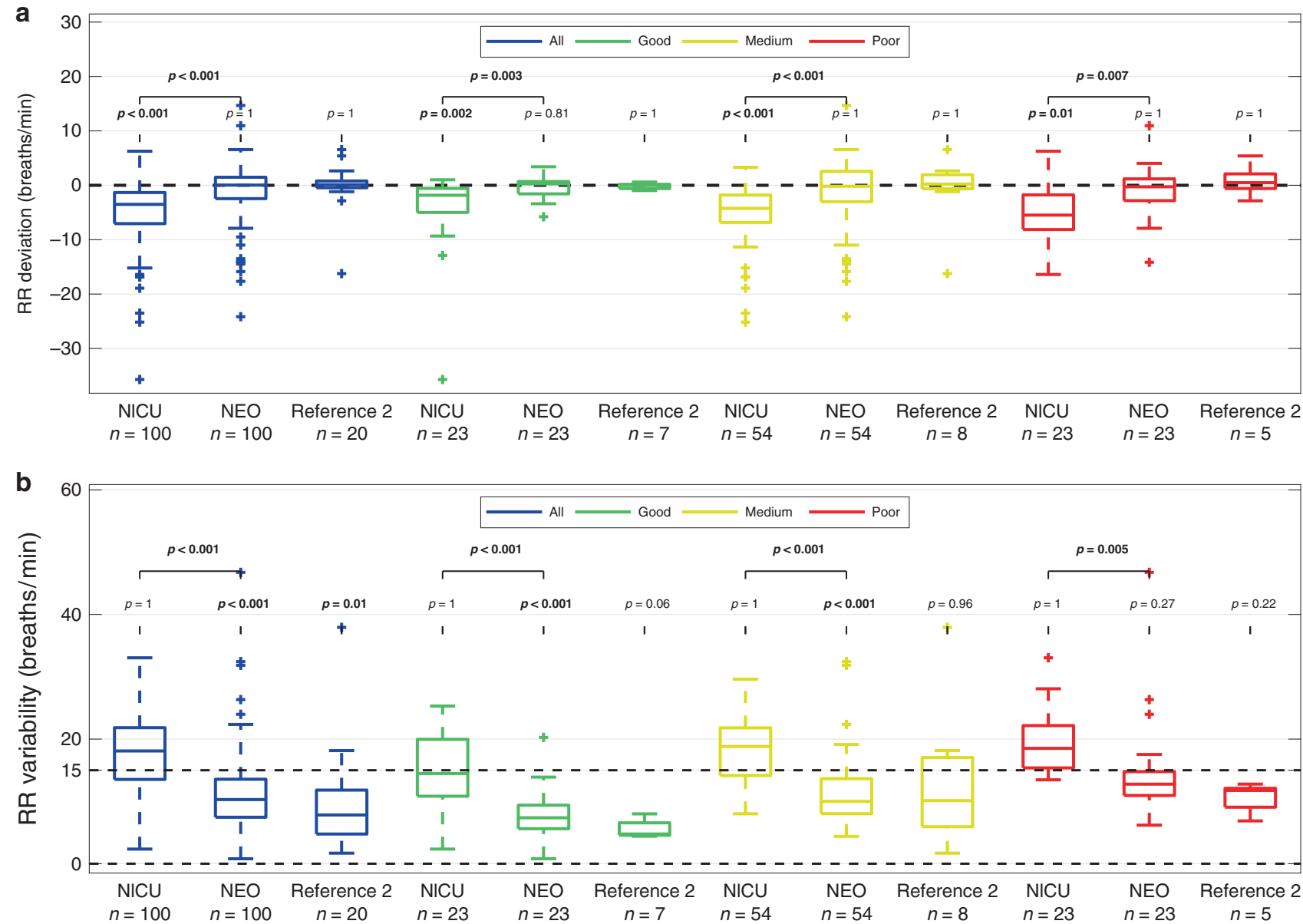

Fig. 4 Comparison of respiratory rate from different sources. $\mathbf{a}$, $\mathbf{b}$ Difference of deviation (median) and variability (interquartile range) of respiratory rate from the reference for different signal source annotation techniques within the segments. Manual labeling of respiratory rate on NEO signal taken as reference, compared to NICU signal using chest impedance, NEO signal analyzed with Kalman filter (NEO), and from Reference 2 (second manual annotation on NEO signal). Displayed as all example episodes (blue, $n=100$ ), subgroup of visually annotated as good esophageal signal quality (green, $n=23$ ), medium signal quality (yellow, $n=54$ ), and of poor signal quality (red, $n=23$ ). Comparison performed using Wilcoxon signed-rank test. a Median deviation of respiratory rate, $\mathbf{b}$ interquartile range variability of respiratory rate. A significantly better agreement between the annotation techniques and the reference, corresponding to significantly lower variability than the set cut-off of 15 breaths per minute (dashed line 15) was considered as clinically relevant. NEO Neonatal Esophageal Observation, NICU standard monitoring from neonatal intensive care unit, RR respiratory rate.

visual labeling vs. Kalman filtering approach $(p=0.81$ for good and 1.0 for medium and poor signal quality). In contrast, significant deviations between visual labeling and NICU RR could be observed irrespective of esophageal signal quality $(p=0.002$ for good, $p<0.001$ for medium, and $p=0.01$ for bad signal quality). RR variability showed significantly better agreement of the NEO Kalman filtering approach with manual labeling within segments of good and medium quality $(p<0.001$, corresponding to a difference lower than the set cut-off of $15 \mathrm{bpm}$ within the segments) and a non-significant difference to $15 \mathrm{bpm}$ variability in segments with poor signal quality $(p>0.05)$. When comparing variability of visually labeled NEO RR and RR derived by NICU, all segments showed differences exceeding the pre-defined cutoff of $15 \mathrm{bpm}$, irrespective of signal quality $(p=1.0$, see also Fig. 4a, b).

\section{Secondary findings}

Inter-rater reliability on visual NEO respiration labeling assessed using Spearman's rank correlation coefficient showed a high correlation with median (IQR) $0.8(0.51-0.87) \mathrm{bpm}$ when looking at all 20 selected segments. Figure 3 of the Supplementary Material also depicts the decreasing agreement between the two raters in case of lower signal quality.

\section{DISCUSSION}

Results from this study indicate that detection of esophagealderived respiration is safe and feasible in non-ventilated preterm infants. The median deviation of RR estimation was $0.04 \mathrm{bpm}$ in periods of clinically meaningful events such as bradycardia and oxygen desaturation and was not considerably altered during periods of low esophageal signal quality.

\section{Interpretation of results}

Automatically extracted RR from NEO signal using an extended Kalman filter is significantly closer to the visually labeled RR in terms of deviation (median values) as well as variability (IQR), than RR derived by NICU. The better agreement was particularly present in segments of good signal quality. These results are confirmed in the Bland Altman plot, with a higher concentration of points near the $x$-axis when comparing NEO than when comparing NICU to the visually labeled RR. Our results indicate a higher robustness of the esophageal method to signal perturbations. Most likely, esophageal electrodes benefit from a more stable electrochemical environment within the esophagus and the proximity to the diaphragm, making them less prone to motion artifacts. Such artifacts resulting from catheter motion, e.g., due to esophageal peristalsis or head movements have not provoked signal 

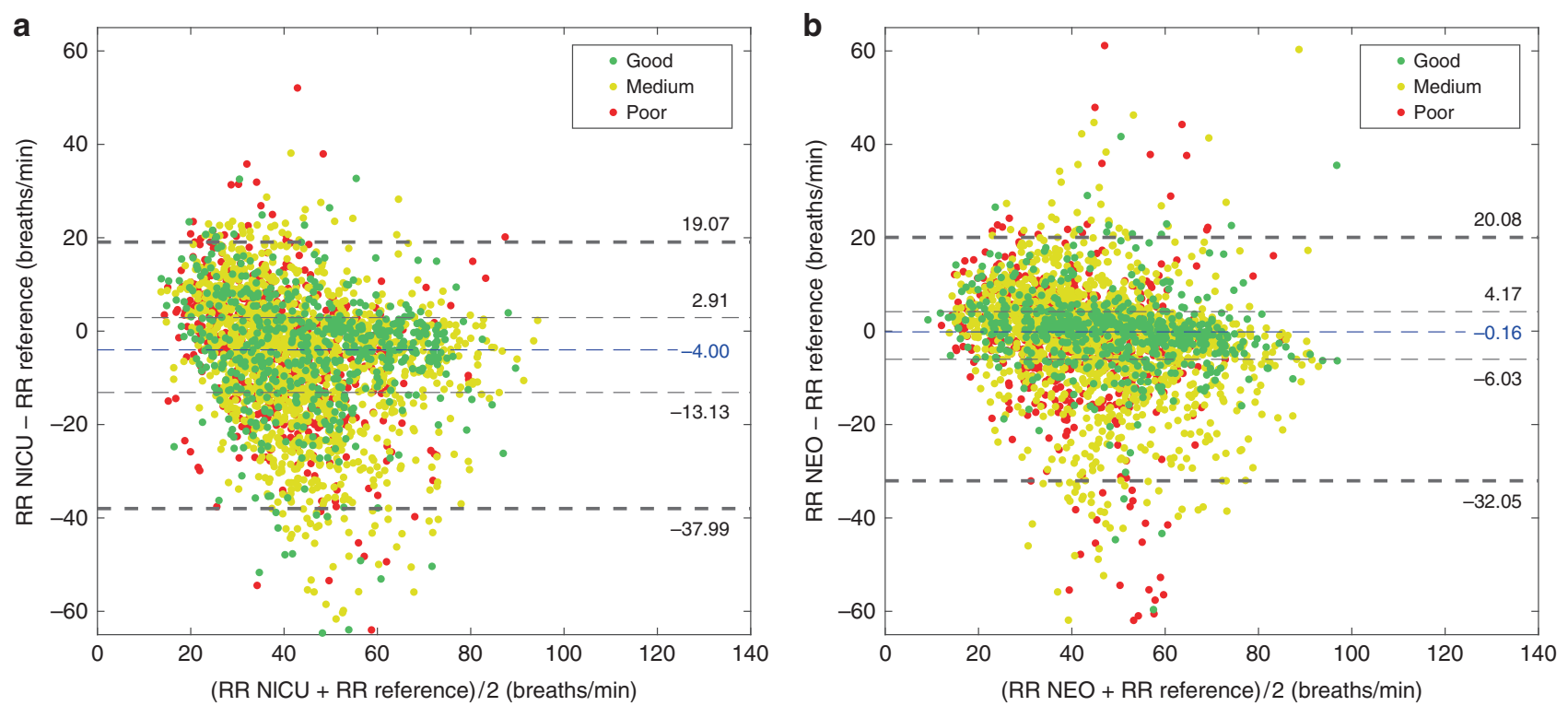

Fig. 5 Bland Altman plot comparing NEO and NICU. a, b Bland Altman plot showing comparison between detecting respiratory rate using Reference (manual annotation of esophageal signal) and NICU (chest impedance from standard monitoring) (a) and NEO (extended Kalman filter based on esophageal signal) (b). Different esophageal signal qualities are colored as green (good), yellow (medium), and red (poor). Blue dashed line indicates median, inner black dashed lines indicate the interquartile range, and outer black dashed lines the $95 \%$ limits of agreement.

saturation due to the DC-coupling amplifier ${ }^{19}$ and thus can subsequently be attenuated by the Kalman filter. In contrast, $\mathrm{Cl}$ derived signals from surface electrodes suffer from artifacts due to skeletal muscle activity or electrode/cable motion. Such artifacts often manifest as saturation of the impedance signal in case of high skeletal muscle activity (see also Fig. 2 of the Supplementary Material), making a reliable detection of RR during those periods cumbersome. This could lead to under-recognition of apnea when relying on $\mathrm{Cl}$ as a signal source.

The association of lower inter-rater reliability and bad signal quality is understandable, given the more difficult task of manual labeling in case of noisy and artifact-rich signals. The fact that esophageal electrodes were only attached over a total length of 6 $\mathrm{cm}$ at the distal end of the Edi tube could lead to already significant signal degradation in case of minor dislocation of the catheter. The extended Kalman filter approach compensated this with its ability to use signal information from all the esophageal channels to extract respiration activity. This estimation is based on "predictions" calculated from the assumed system dynamics (sinusoidal behavior of respiratory activity) and the optimal "corrections" to the estimated states based on the observed values. The weights (the process and measurements noises in the Kalman filter), which determine the extent of these "corrections," are calculated at each time step from the averaged quantities of the past estimations. Indeed, the predictive model helps to suppress abrupt changes in the estimated parameters due to nonstationary signal disturbances, whereas the continuous adjustment of the weights helps in adapting to changes in signal characteristics due to catheter movements.

\section{Comparison with previous literature}

The limited accuracy of today's standard respiratory monitoring on a NICU using $\mathrm{Cl}$ is well known. ${ }^{11}$ Apnea is therefore only indirectly measured, i.e., assumed to be the cause of subsequent events, such as bradycardia or oxygen desaturation. Several research groups recently tried to improve monitoring techniques to better depict the respiratory pattern of newborns. Kraaijenga et al. compared surface diaphragmatic EMG measures with $\mathrm{Cl}$ in infants and found comparable results, with similar susceptibility to surface electrode motions. ${ }^{11}$ Other studies focused on the usability of esophageal signals applying the conventional NAVA interface in both ventilated ${ }^{15,16}$ and non-ventilated preterm infants. ${ }^{18}$ However, a recently published systematic review concludes that the evidence about usefulness of NAVA in infants on non-invasive respiratory support is too limited to draw firm conclusions about its clinical utility yet. ${ }^{17}$

In contrast to the described EMG-based techniques, the applied Kalman filter extracts respiratory activity from diaphragm movement, which manifests as low-frequency baseline wander of the esophageal signal with pronounced magnitudes. Commercially available NAVA respiration detection is based on the EMG of the diaphragm (EMGdi), which usually suffers from low magnitude in the esophageal leads, similar to surface EMG measurements. Reliable detection of esophageal EMGdi is further dependent on the relative position between electrodes and diaphragm, which is difficult to maintain over long observation periods. With our method, we expect the motion signal to be less spatially localized and therefore less dependent on the electrode-diaphragm distance. Besides, the motion signal is easily distinguishable from higher frequency components such as the ECG, EMG from other muscles, and the power-line interference, all turning a reliable detection of EMGdi difficult.

Other research groups are focusing on non-contact monitoring techniques, which would reduce the risk of skin irritation when compared to standard monitoring or surface EMG. Most of these studies, however, are performed in healthy adults or under wellcontrolled research environments during a limited time period, which makes the findings less applicable for continuous monitoring in a NICU. One study performed over several consecutive days in a NICU using high-resolution video-based detection of different vital signs could demonstrate high agreement with standard monitoring for detection of HR. ${ }^{25}$ However, for RR it seemed to be less accurate based on the visual data reported in this study. Unfortunately, the authors did not provide a statistical analysis of the RR data, therefore a statement on accuracy of videomonitoring cannot be made. Besides, during periods of spontaneous movements of infants or lack of visible skin surface, due to, e.g., clothing, instrumentation, shielding of patients, or as a results of humidity in an incubator, vital signs cannot be measured accurately. ${ }^{25}$ Another group used piezoelectric sensors between 
the infants and the mattress to measure an acoustic signal of the cardiorespiratory activity. Again, HR was detected with high robustness and especially well in quiet episodes. RR on the other hand was comparable to standard monitoring during quiet episodes but corrupted during motion or when mechanical ventilator noise disturbed the signal. ${ }^{26}$ The mentioned techniques are promising especially in a controlled research environment but might be difficult to apply in daily clinical practice in a NICU. Especially in critical situations, accurate monitoring is key, but care-givers or instruments might block the view to the body of the patients or introduce noise that disturbs acoustic signals. Although the NEO approach estimates RR from diaphragm motion, the above limitation does not hold as the signal is acquired from sensors incorporated into the body. The multichannel esophageal signals are acquired with electrodes mounted on a gastric feeding tube, a commonly required long-term requisite in preterm infants. Thus, by combining a feeding tube with sensors to detect vital signs, skin irritations and lesions may be mitigated. Some studies used esophageal manometry to estimate respiration activity; however, these did not report any comparison with standard monitoring. ${ }^{27-29}$ Additionally, currently available manometry catheters are too big and inflexible to be used for long-term monitoring and feeding as needed in a NICU.

The usage of tiny skin-interface sensors for wireless measurements of various vital signs was proposed lately in a report of two pilot studies. ${ }^{30}$ The obtained vital signs were not only comparable to standard monitoring techniques but also provided additional information on hemodynamic and cardiovascular health as well as on activity and position of the study participants. ${ }^{30}$ Although the respiration signal was estimated based on surface ECG and skin vibration measurements using electrodes without cables, motion artifacts could not be completely suppressed.

\section{Clinical impact}

Correct detection of critical situations resulting from a prolonged cessation of breathing is of major importance in a NICU. The monitor should detect all clinically relevant episodes and raise as little false positive alarms as possible such that alarm fatigue in health care staff can be prevented..$^{31,32}$ One possible way to reduce false alarms would be to incorporate additional sensor modalities, which, however, until now are still a matter of ongoing research as shown by Muroi et al. ${ }^{33}$ Another attempt would be to improve the primary detection of the signal of interest by reducing noise and artifacts at the sensor side, as we aimed to test with the acquisition of multichannel esophageal signals. The proposed NEO method captures changes of RR instead of indirect measures as bradycardia or desaturation. This direct measure would lead to earlier recognition of potentially dangerous situations. A more accurate respiration signal would also lead to a better understanding of apneas, which could finally improve patient care and consequently reduce health care costs. ${ }^{34,35}$

Esophageal electrodes require the installation of a gastric tube, which bear a minimal risk of local irritation or even infection. If improperly placed, the tube can also affect breathing and HR. Nevertheless, patients in a NICU usually require a feeding tube due to immature swallow-breath coordination anyway and esophageal electrodes do not add any additional risk. The replacement of the surface sensors by esophageal electrodes would additionally decrease the number of wires attached to the patient's body, leading to easier patient care as well as bonding while simultaneously protecting the infant's skin.

However, to date a real-time implementation of RR estimation based on multichannel esophageal signals has not yet been possible but would first have to be tested in a prospective clinical study.

\section{Implication for future research}

Mathematical characteristics of vital signs have been increasingly studied among preterm infants, mainly for its predictive value as marker for early detection of sepsis. ${ }^{36-38}$ The main focus so far has been on HR characteristics, as the activity of the heart is easier to detect with common standard surface monitoring and results are therefore more reliable. Some studies have additionally assessed respiratory patterns, but mainly within short, controlled measurement episodes or selected patient populations, limiting the generalizability of findings in daily clinical practice. ${ }^{39,40}$ Therefore, as reliable data about maturation of breathing (ir)regularity is missing, a lot of physiological knowledge is still to be elaborated. There might be plenty of prognostic physiological information within the breathing pattern of neonates, potentially useful for prediction of both acute and more prolonged outcomes, ${ }^{39,41}$ which is not accessible from today's standard monitoring. This highlights the necessity of an accurate technique to continuously estimate RR in daily clinical practice. We therefore believe that the present study demonstrates a valuable basis for further investigating this research route.

A limitation of $\mathrm{Cl}$ is its inability to detect obstructive apnea. In the current analysis, we did not test to detect obstructive apnea. However, it is conceivable that the changes in diaphragm activity during obstructive apnea manifest as a change of characteristics of motion (e.g., instantaneous amplitude or frequency), which are estimated by the Kalman filter with a high degree of resolution.

Whether the NEO technique can be adopted to even more premature infants or to infants on mechanical ventilation, and whether real-time application would lead to similar results, needs to be tested in future studies. Additionally, improvements of the used catheter, e.g., by enlarging the electrode surface and number of electrodes to cover a wider section of the esophagus, could improve esophageal signal quality and hence the reliability of the method. Also, incorporating more sensing modalities (e.g., pressure and acceleration sensors) into a single gastric catheter can increase the reliability of the estimated parameters. The Kalman filter approach can be extended to include signals from multiple sensors and thus might benefit from the differences in their sensitivities for various physiological processes. Technological advances in the miniaturization of sensors might enable their integration into the NEO catheter extending the diagnostic capability similar to soft skin interfaces. ${ }^{30}$

\section{Strengths and limitations}

A major difficulty when assessing the NEO technique was the lack of a gold-standard to measure RR accurately during daily clinical care in non-ventilated infants given the fact that $\mathrm{Cl}$ is known to be prone to motion artifacts and signal saturation. There are several ways to assess more detailed ventilation parameters in nonventilated infants during study settings using lung function measurements. ${ }^{42}$ But until now these techniques are only applied in controlled research environments and special study settings. We therefore visually inspected and manually labeled NEO signals to assure correct detection by the applied Kalman filter, additionally to the comparison of NEO vs. NICU signals. It must be considered that during manual labeling of NEO signals the NICU respiration signal was visible. Besides, we did perform manual labeling of the esophageal signal and later compared both NEO Kalman and NICU data with this visually labeled method. Both approaches could bias the results.

As the electrodes are placed in the esophagus, interference (e.g., due to peristalsis) with the breathing signal and therefore false high or false low RR is possible. As esophageal peristalsis can be seen as a motion moving from proximal to distal, it affects all electrodes consecutively with a small time shift and usually shows a larger amplitude than respiration activity. We therefore think that this did not affect the visual labeling of RR and hence comparison between NEO and NICU substantially.

A further limitation is the small number of patients included in this study. Given the study design to test safety and feasibility of the NEO technique and the high number of measurement hours, 
as well as clinically relevant episodes that could be studied, we expect that a larger population would not considerably have affected the study results.

\section{Conclusion}

Multichannel esophageal measurement of RR in non-ventilated infants is feasible and safe. Applying an extended Kalman filter to the esophageal signal results in estimation of RR closer to visually annotated RR than that of RR derived from standard $\mathrm{Cl}$ monitoring during meaningful episodes of bradycardia and oxygen desaturation. Thus, esophageal respiration signals might be more reliable due to their proximity to the organ of interest and robustness against motion artifacts or sensor displacements compared to surface electrodes. Applicability of real-time monitoring of RR using multichannel esophageal signals and its non-inferiority compared to standard surface monitoring during daily clinical practice need to be tested in future prospective studies.

\section{REFERENCES}

1. Chawanpaiboon, S. et al. Global, regional, and national estimates of levels of preterm birth in 2014: a systematic review and modelling analysis. Lancet Glob. Health 7, e37-e46 (2019).

2. Martin, R. J. \& Abu-Shaweesh, J. M. Control of breathing and neonatal apnea. Biol. Neonate 87, 288-295 (2005).

3. Abu-Shaweesh, J. M. \& Martin, R. J. Neonatal apnea: what's new? Pediatr. Pulmonol. 43, 937-944 (2008).

4. Poets, C. F., Stebbens, V. A., Samuels, M. P. \& Southall, D. P. The relationship between bradycardia, apnea, and hypoxemia in preterm infants. Pediatr. Res. 34, 144-147 (1993)

5. Eichenwald, E. C. \& Committee on Fetus and Newborn. Apnea of prematurity. Pediatrics 137, e20153757 (2016).

6. Finer, N. N., Barrington, K. J., Hayes, B. J. \& Hugh, A. Obstructive, mixed, and central apnea in the neonate: physiologic correlates. J. Pediatr. 121, 943-950 (1992).

7. Di Fiore, J. M., Poets, C. F., Gauda, E., Martin, R. J. \& MacFarlane, P. Cardiorespiratory events in preterm infants: interventions and consequences. J. Perinatol. 36, 251-258 (2016).

8. Poets, C. F. et al. Association between intermittent hypoxemia or bradycardia and late death or disability in extremely preterm infants. JAMA 314, 595-603 (2015).

9. Di Fiore, J. M. Neonatal cardiorespiratory monitoring techniques. Semin. Neonatol. 9, 195-203 (2004).

10. Di Fiore, J. M., Poets, C. F., Gauda, E., Martin, R. J. \& MacFarlane, P. Cardiorespiratory events in preterm infants: etiology and monitoring technologies. J. Perinatol. 36, 165-171 (2016).

11. Kraaijenga, J. V., Hutten, G. J., de Jongh, F. H. \& van Kaam, A. H. Transcutaneous electromyography of the diaphragm: a cardio-respiratory monitor for preterm infants. Pediatr. Pulmonol. 50, 889-895 (2015).

12. Kraaijenga, J. V. et al. Classifying apnea of prematurity by transcutaneous electromyography of the diaphragm. Neonatology 113, 140-145 (2018).

13. Rutter, N. The newborn skin. Semin Neonatol. 5, 271 (2000).

14. Oranges, T., Dini, V. \& Romanelli, M. Skin physiology of the neonate and infant: clinical implications. Adv. Wound Care 4, 587-595 (2015).

15. Beck, J. et al. Patient-ventilator interaction during neurally adjusted ventilatory assist in low birth weight infants. Pediatr. Res. 65, 663-668 (2009).

16. Stein, H. \& Howard, D. Neurally adjusted ventilatory assist in neonates weighing <1500 grams: a retrospective analysis. J. Pediatr. 160, 786.e1-789.e1 (2012).

17. Goel, D., Oei, J. L., Smyth, J. \& Schindler, T. Diaphragm-triggered non-invasive respiratory support in preterm infants. Cochrane Database Syst. Rev. 3, CD012935 (2020).

18. Sinderby, C. \& Beck, J. Neurally adjusted ventilatory assist in non-invasive ventilation. Minerva Anestesiol. 79, 915-925 (2013).

19. Simmen, P. et al. Multichannel esophageal heart rate monitoring of preterm infants. IEEE Trans. Biomed. Eng. 68, 1903-1912 (2020).

20. Niederhauser, T. et al. Graphics-processor-unit-based parallelization of optimized baseline wander filtering algorithms for long-term electrocardiography. IEEE Trans. Biomed. Eng. 62, 1576-1584 (2015).

21. Niederhauser, T. et al. A baseline wander tracking system for artifact rejection in long-term electrocardiography. IEEE Trans. Biomed. Circuits Syst. 10, 255-265 (2016).
22. Khreis, S., Ge, D., Rahman, H. A. \& Carrault, G. Breathing rate estimation using kalman smoother with electrocardiogram and photoplethysmogram. IEEE Trans. Biomed. Eng. 67, 893-904 (2020).

23. McNames, J. \& Aboy, M. Cardiovascular signal decomposition and estimation with the extended Kalman smoother. Conf. Proc. IEEE Eng. Med Biol. Soc. 2006, 3708-3711 (2006).

24. Vullings, R., de Vries, B. \& Bergmans, J. W. An adaptive Kalman filter for ECG signal enhancement. IEEE Trans. Biomed. Eng. 58, 1094-1103 (2011).

25. Villarroel, M. et al. Continuous non-contact vital sign monitoring in neonatal intensive care unit. Healthc. Technol. Lett. 1, 87-91 (2014).

26. Sato, S. et al. Assessment of a new piezoelectric transducer sensor for noninvasive cardiorespiratory monitoring of newborn infants in the NICU. Neonatology 98, 179-190 (2010).

27. Welty, S. E. et al. Short term evaluation of respiratory effort by premature infants supported with bubble nasal continuous airway pressure using seattle-pap and a standard bubble device. PLOS ONE 13, e0193807 (2018).

28. Rayyan, M., Omari, T., Debeer, A., Allegaert, K. \& Rommel, N. Characterization of esophageal motility and esophagogastric junction in preterm infants with bronchopulmonary dysplasia. Neurogastroenterol. Motil. 32, e13849 (2020).

29. Essouri, S. et al. Relationship between diaphragmatic electrical activity and esophageal pressure monitoring in children. Pediatr. Crit. Care Med. 20, e319-e325 (2019).

30. Chung, H. U. et al. Skin-interfaced biosensors for advanced wireless physiological monitoring in neonatal and pediatric intensive-care units. Nat. Med. 26, 418-429 (2020).

31. Brockmann, P. E. et al. Under-recognition of alarms in a neonatal intensive care unit. Arch. Dis. Child. Fetal Neonatal Ed. 98, F524-F527 (2013).

32. Joshi, R. et al. Pattern discovery in critical alarms originating from neonates under intensive care. Physiol. Meas. 37, 564-579 (2016).

33. Muroi, C. et al. Automated false alarm reduction in a real-life intensive care setting using motion detection. Neurocrit. Care 32, 419-426 (2020).

34. Eichenwald, E. C. et al. Variation in diagnosis of apnea in moderately preterm infants predicts length of stay. Pediatrics 127, e53-e58 (2011).

35. Zupancic, J. A., Richardson, D. K., O'Brien, B. J., Eichenwald, E. C. \& Weinstein, M. C. Cost-effectiveness analysis of predischarge monitoring for apnea of prematurity. Pediatrics 111, 146-152 (2003).

36. Griffin, M. P. et al. Abnormal heart rate characteristics preceding neonatal sepsis and sepsis-like illness. Pediatr. Res. 53, 920-926 (2003).

37. Moorman, J. R. et al. Mortality reduction by heart rate characteristic monitoring in very low birth weight neonates: a randomized trial. J. Pediatr. 159, 900.e1-906.e1 (2011).

38. Masino, A. J. et al. Machine learning models for early sepsis recognition in the neonatal intensive care unit using readily available electronic health record data. PLOS ONE 14, e0212665 (2019).

39. Navarro, X., Porée, F. \& Beuchée, A. Artifact rejection and cycle detection in immature breathing: application to early detection of neonatal sepsis. Biomed. Signal Process. Control 16, 9-16 (2015).

40. Patel, M. et al. Clinical associations with immature breathing in preterm infants: Part 2-Periodic breathing. Pediatr. Res. 80, 28-34 (2016).

41. Jost, K. et al. Sigh-induced changes of breathing pattern in preterm infants. Physiol. Rep. 3, e12613 (2015).

42. Frey, U. Clinical applications of infant lung function testing: does it contribute to clinical decision making? Paediatr. Respir. Rev. 2, 126-130 (2001).

\section{ACKNOWLEDGEMENTS}

We thank Michaela Verling for her help in data description and analysis.

\section{AUTHOR CONTRIBUTIONS}

Substantial contributions to conception and design: A.H., S.M.S., D.T., T.N., K.J. Substantial contributions to acquisition of data, analysis, and interpretation of data: $C$. B., P.S., N.G., L.S., S.K., S.M.S., T.N., K.J. Writing of first manuscript draft: C.B., N.G., T.N., K. J. Critical revision of the article: P.S., L.S., S.K., S.M.S., D.T., A.H.

\section{FUNDING}

Open Access funding provided by Universität Basel (Universitätsbibliothek Basel). This study was financially supported by the Bangerter-Rhyner Foundation, the European Society of Pediatric Research, the University Children's Hospital Basel, and the University of Basel, Switzerland. None of the supporters had any influence on design or conductance of the study nor on decision about or content of the publication. 


\section{COMPETING INTERESTS}

The authors declare no competing interests.

\section{ADDITIONAL INFORMATION}

Supplementary information The online version contains supplementary material available at https://doi.org/10.1038/s41390-021-01748-4.

Correspondence and requests for materials should be addressed to Kerstin Jost.

Reprints and permission information is available at http://www.nature.com/reprints

Publisher's note Springer Nature remains neutral with regard to jurisdictional claims in published maps and institutional affiliations.
Open Access This article is licensed under a Creative Commons Attribution 4.0 International License, which permits use, sharing, adaptation, distribution and reproduction in any medium or format, as long as you give appropriate credit to the original author(s) and the source, provide a link to the Creative Commons license, and indicate if changes were made. The images or other third party material in this article are included in the article's Creative Commons license, unless indicated otherwise in a credit line to the material. If material is not included in the article's Creative Commons license and your intended use is not permitted by statutory regulation or exceeds the permitted use, you will need to obtain permission directly from the copyright holder. To view a copy of this license, visit http://creativecommons. org/licenses/by/4.0/.

(c) The Author(s) 2021 